\title{
Quantum Frequency Conversion by Four-wave Mixing Using Bragg Scattering
}

Andersen, Lasse Mejling; Rottwitt, Karsten; McKinstrie, C. J. ; Raymer, M. G.

Published in:

Advanced Photonics Congress

Publication date:

2012

Document Version

Publisher's PDF, also known as Version of record

Link back to DTU Orbit

Citation (APA):

Andersen, L. M., Rottwitt, K., McKinstrie, C. J., \& Raymer, M. G. (2012). Quantum Frequency Conversion by Four-wave Mixing Using Bragg Scattering. In Advanced Photonics Congress (pp. JTu5A.18). Optical Society of America.

\section{General rights}

Copyright and moral rights for the publications made accessible in the public portal are retained by the authors and/or other copyright owners and it is a condition of accessing publications that users recognise and abide by the legal requirements associated with these rights.

- Users may download and print one copy of any publication from the public portal for the purpose of private study or research.

- You may not further distribute the material or use it for any profit-making activity or commercial gain

- You may freely distribute the URL identifying the publication in the public portal

If you believe that this document breaches copyright please contact us providing details, and we will remove access to the work immediately and investigate your claim 


\title{
Quantum Frequency Conversion by Four-wave Mixing Using Bragg Scattering
}

\author{
L. Mejling, ${ }^{1, *}$ K. Rottwitt, ${ }^{1}$ C. J. McKinstrie, ${ }^{2}$ and M. G. Raymer, ${ }^{3}$ \\ ${ }^{1}$ Department of Photonics Engineering, Technical University of Denmark, DK-2800 Kgs. Lyngby, Denmark \\ ${ }^{2}$ Bell Laboratories, Alcatel-Lucent, Holmdel, New Jersey 07733, USA \\ ${ }^{3}$ Department of Physics, University of Oregon, Eugene, Oregon 97403, USA \\ *Iman@fotonik.dtu.dk
}

\begin{abstract}
Two theoretical models for frequency conversion (FC) using nondegenerate four-wave mixing are compared, and their range of validity are discussed. Quantum-statepreserving $\mathrm{FC}$ allows for arbitrary reshaping of states for an appropriate pump selection.
\end{abstract}

(C) 2012 Optical Society of America

OCIS codes: (190.4380) Nonlinear optics; four-wave mixing; (270.5585) Quantum information and processing.

\section{Summary}

Quantum communication is becoming increasingly important, and an effective method for reshaping and frequency converting (FC) quantum states is essential [1,2]. Applications include sending a state between nodes (in the $1310 \mathrm{~nm}$ or $1550 \mathrm{~nm}$ communication window) and detecting the state at a shorter wavelength, or reshaping the temporally wide output from quantum memories to a shorter pulse for better utilization of the existing optical infrastructure [3,4].

One possible way to FC quantum states while preserving their quantum properties is by four-wave mixing (FWM) in an optical fiber using the nondegenerate process called Bragg scattering (BS), where two strong pumps $\mathrm{p}$ and $\mathrm{q}$ interact with two sidebands $\mathrm{r}$ and s, such that $\pi_{\mathrm{p}}+\pi_{\mathrm{s}} \leftrightarrow \pi_{\mathrm{q}}+\pi_{\mathrm{r}}$, where $\pi_{j}$ is a photon at the frequency $\omega_{j}, j \in\{\mathrm{p}, \mathrm{q}, \mathrm{r}, \mathrm{s}\}[5,6]$.

We describe FC by BS by the coupled mode equations (CMEs)

$$
\left(\partial_{z}+\beta_{\mathrm{r}} \partial_{t}\right) A_{\mathrm{r}}=\mathrm{i} \gamma_{\mathrm{pq}} A_{\mathrm{s}}, \quad\left(\partial_{z}+\beta_{\mathrm{s}} \partial_{t}\right) A_{\mathrm{s}}=\mathrm{i} \gamma_{\mathrm{pq}}^{*} A_{\mathrm{r}},
$$

where $\beta_{\mathrm{r}}$ and $\beta_{\mathrm{s}}$ are the group slownesses (inverse group velocities) of the idler and the signal respectively, and $A_{\mathrm{r}}(t, z)$ and $A_{\mathrm{s}}(t, z)$ are the sideband amplitudes whose squares are in units of power. The coefficient $\gamma$ is proportional to the Kerr nonlinearity coefficient, and for waves that are not too far separated in frequency $\beta_{\mathrm{s}}=\beta_{\mathrm{p}}$ and $\beta_{\mathrm{q}}=\beta_{\mathrm{r}}$ [6]. The coupling function is $\gamma_{\mathrm{pq}}=\gamma A_{\mathrm{p}}\left(t-\beta_{\mathrm{s}} z\right) A_{\mathrm{q}}^{*}\left(t-\beta_{\mathrm{r}} z\right)$, where the pump shapes are normalized. For simplicity we only consider the Green function (GF) describing the influence on the output idler s from the input signal r, $G_{\mathrm{rs}}$. In the low-efficiency limit, $\gamma /\left|\beta_{\mathrm{r}}-\beta_{\mathrm{s}}\right|=\gamma / \beta_{\mathrm{rs}}=\bar{\gamma} \ll 1$, one sideband is assumed constant leading to the perturbative solution

$$
G_{\mathrm{rs}}^{\text {pert. }}\left(t ; t^{\prime}\right)=\mathrm{i} \bar{\gamma} A_{\mathrm{q}}^{*}\left[t-\beta_{\mathrm{r}}\left(l-z_{\mathrm{i}}\right)\right] A_{\mathrm{p}}\left[t^{\prime}+\beta_{\mathrm{s}} z_{\mathrm{i}}\right] \mathrm{H}\left(t^{\prime}+\beta_{\mathrm{r}} l-t\right) \mathrm{H}\left(t-t^{\prime}-\beta_{\mathrm{s}} l\right),
$$

where $\mathrm{H}$ is the Heaviside step-function and $z_{\mathrm{i}}$ the interaction distance. A remarkable consequence of this result is that for long fibers the step-functions tend to unity and the GF is separable in $t$ and $t^{\prime}$. Using standard mathematical methods the CMEs are solvable for an arbitrary strength parameter leading to the exact solution

$$
G_{\mathrm{rs}}^{\text {exact }}\left(t ; t^{\prime}\right)=\mathrm{i} \bar{\gamma} A_{\mathrm{q}}^{*}(t, l) \mathrm{J}_{0}\left\{2 \bar{\gamma}\left[\xi\left(t, t^{\prime}\right) \eta\left(t, t^{\prime}\right)\right]^{1 / 2}\right\} A_{\mathrm{p}}\left(t^{\prime}, 0\right) \mathrm{H}\left(t^{\prime}+\beta_{\mathrm{r}} l-t\right) \mathrm{H}\left(t-t^{\prime}-\beta_{\mathrm{s}} l\right),
$$

where $\mathrm{J}_{0}$ is the Bessel function of zeroth order and the effective interaction distances are $\eta\left(t, t^{\prime}\right)=\int_{t^{\prime}}^{t-\beta_{s} l}\left|A_{\mathrm{p}}(s)\right|^{2} \mathrm{~d} s$ and $\xi\left(t, t^{\prime}\right)=\int_{t-\beta_{\mathrm{r}} l}^{t^{\prime}}\left|A_{\mathrm{q}}(s)\right|^{2} \mathrm{~d} s$. Equations (2) and (3) are valid for arbitrary pump shapes.

The interesting parameter that differentiates the exact GF (3) from the perturbative GF (2), is the strength parameter $\bar{\gamma}$. In the low-efficiency regime the two models agree, whereas for larger values a qualitative different behavior is observed which is confirmed in Fig. 1(a) and (b). The perturbative GF scales linearly with $\bar{\gamma}$, while the exact GF exhibits a complex dependence in $\bar{\gamma}$. The Schmidt coefficients are the square roots of the singular values of the GF [6, OE]. They are useful as they predict separability, and from Fig. 1(c) we see that the perturbative GF is separable in this 


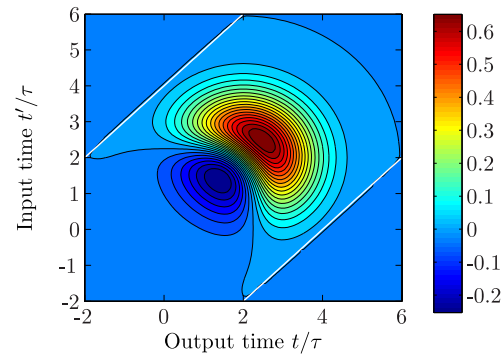

(a)

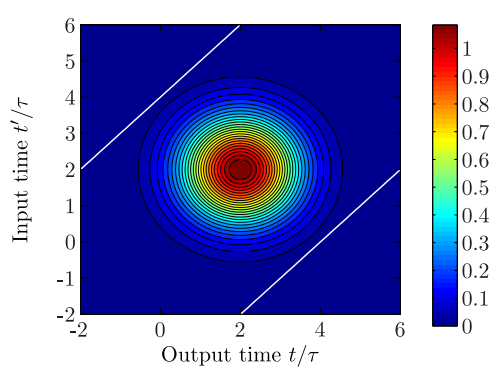

(b)

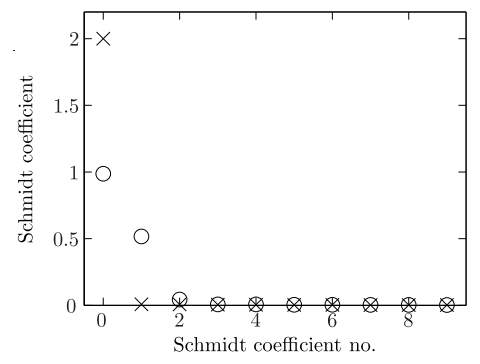

(c)

Fig. 1. Plots in the high-conversion regime $\bar{\gamma}=2$ and $\beta l / \tau=4$. The white lines mark the area of causality. (a) Contour plot of the exact GF. (b) Plot of the perturbative GF. (c) The first ten Schmidt coefficients, crosses denote the approximate GF.

limit, in contrast to the fact that we have more than one nonzero Schmidt coefficient. All the results are for Gaussian pumps and more interesting pump shapes will be discussed at the meeting.

To quantify the difference between the two GFs, the ratio $\iint\left|G_{\mathrm{rs}}^{\text {exact }}-G_{\mathrm{rs}}^{\text {pert. }}\right|^{2} \mathrm{~d} t \mathrm{~d} t^{\prime} / \iint\left|G_{\mathrm{rs}}^{\text {exact }}\right|^{2} \mathrm{~d} t \mathrm{~d} t^{\prime}$ was calculated, see Fig. 2(a). For all the considered pump shapes, less than $5 \%$ deviation is possible for $\bar{\gamma}<0.7$. Consider one particular photonic crystal fiber with $\beta_{\mathrm{rs}} \sim 10^{-11} \mathrm{~s} \mathrm{~m}^{-1}$ this means that the perturbative solution is valid for pump energies of the order $10^{-8} \mathrm{~J}$ [6]. Figure 2(b) shows the square of the two lowest-order squared Schmidt coefficients for the exact Green function and the squared lowest-order coefficient for the perturbative model. Figure 2(b) does not depend on the pump shape but is general as long as a complete collision has occurred. For $\bar{\gamma}>1$ complete separability is not possible in the exact model, but this is not captured by the simpler one as it only has one nonzero Schmidt coefficient. However, for $95 \%$ conversion efficiency $\left(\lambda_{0}\right)$ in the exact model, the first coefficient is nine times larger than the second one, so it is approximately separable. For $\bar{\gamma}=0.7$ a conversion efficiency of $35 \%$ is achievable.

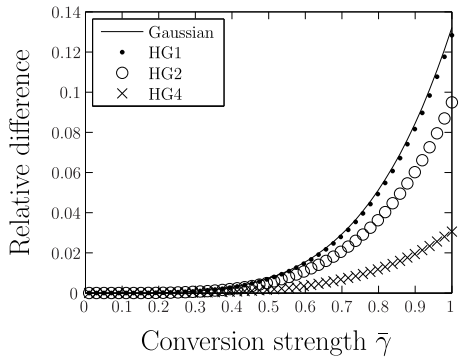

(a)

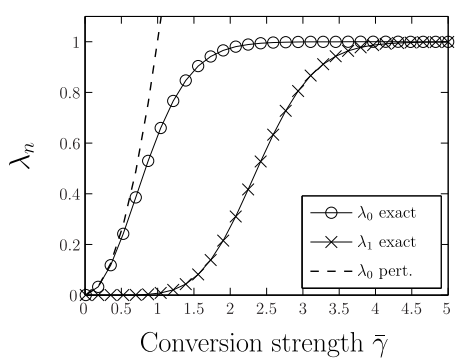

(b)

Fig. 2. (a) The relative difference between the two models for $\beta l / \tau=4$ for different pumps where HG $n$ is Hermite-Gauss modes of order $n$. (b) The square of the Schmidt coefficients for HG0 pumps.

In summary, we showed that the exact solution for frequency-conversion using Bragg scattering describes effects that are not captured by the simpler model, but a good agreement between the two models is possible for $\bar{\gamma}<0.7$. The problem of self-phase and cross-phase modulation will be considered elsewhere. These effects chirp the Schmidt modes, but their power profiles are similar as those predicted by Eqs. (2) and (3) [6, OE].

\section{References}

1. S. Tanzilli et al., Nature (London) 437, 116-20 (2005).

2. B. Brecht et al., New J. Phys. 13, 065029 (2011).

3. W. Wasilewski and M. G. Raymer, Phys. Rev. A 73, 063816 (2006).

4. C. Clausen et al., Nature (London) 469, 508-511 (2011).

5. C. J. McKinstrie et al., Opt. Express 13, 9131-9142 (2005).

6. H. J. McGuinness et al., Phys. Rev. Lett. 105, 093604 (2010), Opt. Express (OE) 19, 17876-17907 (2011). 